$55 \mid 2015$

(Se) former pour enseigner le français à ceux qui ne le parlent pas nativement -

\title{
Se former pour enseigner ou enseigner pour se former ? Formations et parcours d'enseignants en Grèce au XIXe siècle
}

\section{Despina Provata}

\section{(2) OpenEdition}

\section{Journals}

Édition électronique

URL : https://journals.openedition.org/dhfles/4293

DOI : $10.4000 /$ dhfles.4293

ISSN : 2221-4038

Éditeur

Société Internationale pour l'Histoire du Français Langue Étrangère ou Seconde

Édition imprimée

Date de publication : 1 décembre 2015

Pagination : 101-119

ISSN : 0992-7654

Référence électronique

Despina Provata, «Se former pour enseigner ou enseigner pour se former? Formations et parcours d'enseignants en Grèce au XIXe siècle », Documents pour I'histoire du français langue étrangère ou seconde [En ligne], 55 | 2015, mis en ligne le 01 janvier 2018, consulté le 28 mars 2023. URL : http:// journals.openedition.org/dhfles/4293 ; DOI : https://doi.org/10.4000/dhfles.4293

Ce document a été généré automatiquement le 28 mars 2023.

Tous droits réservés 


\title{
Se former pour enseigner ou enseigner pour se former? Formations et parcours d'enseignants en Grèce au XIXe siècle
}

\author{
Despina Provata
}

1 La Grèce, à l'exemple d'autres états européens qui lancent au XIX ${ }^{\mathrm{e}}$ siècle une vaste entreprise de réforme de l'enseignement secondaire au cœur de laquelle figurent les langues vivantes, va progressivement procéder à l'institutionnalisation du français langue vivante comme discipline scolaire. Ce mouvement s'amorce alors même que la guerre d'Indépendance, déclenchée en 1821, bat son plein. En 1822, la nécessité d'introduire l'enseignement $d u$ français dans les écoles est énoncée de manière solennelle par le Sénat du Péloponnèse ${ }^{1}$ : dans un texte qui porte le titre explicite Manifeste de toute administration éclairée la Chambre haute invite « des maîtres habiles » à enseigner, entre autres matières, la langue française (Dimaras 1973: 4-5). Or, aucune structure étatique n'est alors prévue à cet effet.

2 La présente étude se propose d'examiner la question de la formation des maitres et celle des professeurs de français au cours du XIX ${ }^{e}$ siècle. Quels textes ou déclarations traitent-ils de la question et dans quelle mesure ont-ils été suivis ? Mais avant d'essayer de répondre à ces interrogations concernant les différentes formations, les modalités de recrutement et les différents parcours des professeurs de français en Grèce au XIX ${ }^{e}$ siècle, il est utile de rappeler brièvement d'un côté la place du français dans l'enseignement secondaire grec, et de l'autre l'occasion manquée de créer une chaire universitaire de langue et littérature françaises. 


\section{Le système scolaire grec et la place du français dans l'enseignement}

3 Après les premières tentatives de Ioannis Capodistrias pour organiser l'enseignement national entre 1828-1832 (Bambounis 1999) et la loi de 1834 qui organisait l'enseignement élémentaire ${ }^{2}$, l'enseignement secondaire est institutionnalisé par un décret royal de 1836. Celui-ci prévoyait deux cycles d'études : trois premières années de scolarité dans l'« École hellénique » et quatre ans de scolarité dans les «Gymnases » (Journal officiel (J.0.), 31-12-1836/12-1-1837). La langue française figure au programme des deux degrés de l'enseignement secondaire et se voit même accorder une place de choix parmi les autres matières du programme ${ }^{3}$. Elle est en effet présente dans les programmes des écoles helléniques où se faisait l'initiation à la langue française, complétée par des exercices de lecture et de récitation. L'enseignement était assuré par le maitre d'école, à l'exception des écoles d'Athènes pour lesquelles étaient prévus des postes d'enseignants spécialisés. Pour les Gymnases, c'est un enseignant spécialisé qui se chargeait de l'approfondissement de la langue à l'aide des chrestomathies selon la méthode traditionnelle (grammaire-traduction).

4 L'institutionnalisation de l'enseignement du français va ainsi conduire à la naissance d'un nouveau corps d'enseignants, recruté et inspecté par l'État. Signalons toutefois, que ce nouveau corps coexiste d'une part avec des précepteurs particuliers dont le nombre ne cesse d'augmenter au long du $\mathrm{XIX}^{\mathrm{e}}$ siècle ${ }^{4}$, et d'autre part avec les enseignants congréganistes, ces ensembles n'étant pas toujours étanches.

\section{La formation et le recrutement des enseignants}

5 Pour cette première génération d'enseignants, aucune qualification spéciale n'est exigée d'après le décret relatif au règlement des Écoles helléniques et des Gymnases. Les maîtres d'école, auxquels on demandait des notions de français, devaient tout simplement prouver leur aptitude devant une commission constituée par le directeur et tous les professeurs du Gymnase. La loi exigeait en outre que pour être titularisés ils devaient "avoir une formation pratique dans l'enseignement soit dans une École hellénique soit dans un établissement privé où ces matières sont enseignées » (J.0., $\mathrm{n}^{\circ}$ 87, 31-12-1836/12-1-1837). Un examen, oral et écrit, devait pouvoir vérifier leur niveau de français. Comme on peut le constater, c'est l'expérience exigée par la loi qui est appelée à combler les lacunes dues à l'absence de formation. Les professeurs de Gymnase, eux, devaient avoir effectué des études universitaires à la "Faculté des sciences générales » et avoir réussi une épreuve écrite et orale devant une commission nommée par le secrétariat des Cultes et de l'instruction (ibid.).

\section{L'occasion manquée de créer une chaire universitaire de langue et littérature françaises}

Les textes de loi attestent donc du souci que les législateurs avaient d'adopter un cadre législatif pour la formation professionnelle des futurs enseignants de français. Il restait cependant à mettre en place les structures nécessaires. 
7 En 1837 est fondée l'Université othonienne (du nom du roi Othon qui fut l'instigateur de sa création), précurseur de l'Université nationale et capodistrienne d'Athènes. Elle était constituée de quatre facultés. La première, nommée «Faculté des sciences générales $»^{5}$, comportait la philosophie, la philologie, les mathématiques et les sciences naturelles, la chimie, la physique, l'astronomie, les différents domaines de l'histoire naturelle, la géographie et l'histoire, ainsi que leurs sciences auxiliaires, sans que ces sciences ne soient toutefois nommées (J.0., $\left.\mathrm{n}^{\circ} 86,31-12-1836 / 21-1-1837\right)$. Peu après, sont publiées dans le J.O. les nominations des premiers professeurs d'université. On y trouve un poste de professeur extraordinaire ${ }^{6}$ de langue et littérature françaises attribué à Progin Soladam, un habitué du palais royal dont on ignore les antécédents, voire la formation. Figure étrange, il se voulait en tout cas réformateur dans le domaine de l'éducation et s'était donné pour mission «la propagation du goût des arts et des sciences non seulement en Europe, mais encore dans les autres parties du monde» ([Soladam] $1831: 4$ ).

8 Lorsqu'un nouveau décret royal, en avril 1837, révise certaines dispositions de la loi précédente et limite les postes d'enseignants au strict nécessaire pour la mise en œuvre de l'Université othonienne, le poste de professeur de français disparaît (J.0. $\mathrm{n}^{\circ} 16$, 24-4-1837)7. Or, ce même Progin Soladam, qui n'a pas abandonné son rêve de devenir professeur à l'université, réitère, en octobre 1837 , sa demande de nomination auprès du secrétariat des Cultes. Une copie de la brève réponse du secrétariat des Cultes par laquelle sa demande est définitivement rejetée constitue le seul document officiel dont nous disposons expliquant les raisons pour lesquelles le projet de création d'une chaire de français fut alors abandonné. En voici la teneur, en français dans l'original : «la chaire de la langue et de la littérature française n'existe pas dans l'Université et [...] même lorsque cet établissement aura atteint son plus haut point de développement, on ne pourra y erriger (sic) des chaires distinctes pour la littérature de chaque pays à part, et encore moins pour les langues vivantes. Une seule chaire de la littérature moderne en comprendra alors toutes les branches " (GAK 1833-1848, série 002, dossier 1517 : Progin Soladam $)^{8}$. La politique invoquée dans cette lettre clôt de cette manière le chapitre à peine ouvert de la création d'une chaire de langue et littérature françaises à l'université. Il ne sera ré-ouvert qu'en $1954^{9}$.

$9 \mathrm{Si}$, pendant plus d'un siècle, il n'existera aucune structure publique pour la formation des enseignants, des transformations lentes et progressives s'amorcent néanmoins vers le milieu du XIX ${ }^{e}$. Elles se concrétisent par une tentative plus systématique de la part de l'État d'établir des procédures précises pour la sélection et la nomination des enseignants de l'enseignement secondaire.

\section{Modalités de recrutement}

10 Ainsi, à partir de 1868 , les candidats aux postes de professeur sont tenus de présenter diplômes ou certificats d'études, ou bien, le cas échéant, de passer un examen d'aptitude devant une commission d'experts (J.0., $\left.\mathrm{n}^{\circ} 39,30-8-1868\right)$. Cette procédure, ouverte aussi bien aux Grecs qu'aux étrangers, est censée pouvoir satisfaire les besoins sans cesse croissants pour un personnel enseignant de haut niveau. Or, en réalité, elle ne fait que révéler la pénurie d'enseignants spécialisés, ce qui oblige le ministère à modifier les dispositions de la loi. De la sorte, on finit par autoriser, en 1885, là où il n'y a pas de professeur spécialisé, qu'un autre enseignant du même ou d'un autre Gymnase 
assure le cours de français (J.0., $\mathrm{n}^{\circ}$ 87, 31-7-1885: 275-276). Il est à noter que cette révision à la baisse des qualifications requises pour les professeurs de français vient un an après la réforme du programme scolaire de 1884 qui fixait de manière détaillée les matières enseignées pour chaque classe (Antoniou 1987 : 244-255).

Cette décision suscita bien évidemment des réactions, car si l'enseignement du français par des professeurs spécialisés n'avait jusqu'alors donné que des résultats médiocres, on ne pouvait que redouter les conséquences d'une telle décision: «si, Messieurs, l'expérience a jusqu'à présent prouvé que l'enseignement de la langue française même par des professeurs spécialisés est très peu fécond, - proteste un député lors de la discussion dans la Chambre -, on peut sans difficulté prévoir à quel point il peut se révéler stérile s'il est confié au professeur d'instruction religieuse ou à celui de mathématiques-physique » (cité dans Antoniou 2012 : 70, note 8).

Ces normes seront de nouveau révisées : quelques mois plus tard, le ministère revient à la charge au sujet de la procédure des examens de qualification. Désormais, pour pouvoir être nommé dans les Gymnases, le candidat devra posséder un diplôme universitaire, grec ou étranger, ou à défaut, un baccalauréat littéraire français. Il devra en outre réussir une double épreuve d'aptitude à l'enseignement $d u$ français mais aussi du grec moderne (J.0., $\mathrm{n}^{\circ}$ 1, 2-1-1886). Notons ici que les candidats français, même lorsqu'ils possédaient un diplôme, n'étaient pas dispensés des épreuves écrites puisque la bonne connaissance du grec moderne était toujours une condition préalable à la titularisation.

\section{Les commissions et les épreuves d'évaluation}

Les commissions d'évaluation et de nomination des futurs enseignants demeurent en réalité tout le long du XIX ${ }^{e}$ siècle la seule instance officielle susceptible de se prononcer sur la formation, la capacité et les talents pédagogiques des futurs enseignants. Nommées par le ministère de l'Instruction, leur composition était variable : on y trouve des professeurs d'université ${ }^{10}$, des érudits et auteurs de manuels de français ${ }^{11}$ mais aussi des professeurs de Gymnase, dont des Français ${ }^{12}$. Ce n'est qu'en 1892 que leur composition sera arrêtée par une loi : les commissions devront dès lors comporter un directeur de Gymnase, un professeur de grec et un professeur de français (J.O., fasc. A, $\left.\mathrm{n}^{\circ} 77,12-3-1892\right)$. Malgré l'absence de procédures uniformes de sélection (et de nomination), ces commissions constituent le seul cadre légal pour l'activité enseignante. Elles témoignent, tant bien que mal, de la volonté de l'État de mettre en place un enseignement de qualité et d'instituer un mécanisme de contrôle.

Pour ce qui est du contenu même des épreuves, aucune loi, aucun texte administratif ne le définissait. Il revenait donc à ces jurys de définir le mode de contrôle des connaissances des candidats. Se pliant aux exigences pédagogiques de l'époque et de la méthode traditionnelle, le jury dictait au candidat un texte en français - dont on ignore aussi bien la teneur que la longueur - qu'il devait ensuite traduire en grec. L'épreuve écrite comportait aussi un exercice de thème. Il n'a pas été jusqu'à présent possible de repérer dans les archives des traces des textes donnés à traduire, mais si l'on tient compte des contenus des programmes scolaires on peut supposer, sans trop de risque d'erreur de notre part, que c'étaient des extraits d'auteurs classiques ${ }^{13}$. L'épreuve était couronnée par un examen oral visant à vérifier la bonne prononciation du candidat. À partir de 1892, s'ajoute aux épreuves écrite et orale des langues grecque et française, 
une épreuve d'histoire, matière qui était souvent enseignée par les professeurs de français. Ainsi, au tournant du $\mathrm{XX}^{\mathrm{e}}$ siècle, personne ne semble encore estimer utile d'ajouter une leçon pratique à l'épreuve ou, à défaut, de détailler les critères de sélection. On comprend alors mieux pourquoi les évaluations des commissions sur la formation et les aptitudes des enseignants sont souvent générales, vagues et abstraites ${ }^{14}$.

Cependant, une attention particulière est accordée à la bonne connaissance du grec moderne car celui-ci est jugé nécessaire pour pouvoir «traduire avec grâce et précision» (GAK 1848-1854, dossier 100/1, fo 5421). Cette partie de l'épreuve est d'ailleurs le point faible des candidats français qui se voient souvent recalés à cause de leur connaissance imparfaite du grec. Il n'est du reste pas rare de voir des professeurs français une fois nommés, susciter les plaintes des élèves, de leurs parents et de leurs supérieurs du fait de ne pas pouvoir communiquer avec leur classe. Parmi ceux-là, citons un Français nommé Loque qui provoqua de nombreuses réactions au gymnase de Nauplie, puis à celui de Syra. Mis à part les problèmes de discipline, signalés de manière réitérée dans les rapports des deux proviseurs, il se heurtait à un réel problème de méthode dans l'enseignement du français car du fait de ne pas parler la langue grecque, il ne pouvait pas appliquer la méthode grammaire-traduction. «Il explique les mots inconnus par d'autres mots français» s'indigne le proviseur du Gymnase (GAK 1848-1854, dossier 82 : Loque).

Parallèlement, la bonne prononciation du français, autrement dit l'articulation correcte, préoccupe beaucoup les jurys: des candidats sont refusés quand la prononciation n'est pas suffisamment claire. Quant à la vérification des connaissances grammaticales, on ignore comment elle se faisait, mais il fallait au moins prouver de les posséder suffisamment. Les candidats devaient connaître le français « dans la pratique et en théorie et maîtriser des règles grammaticales et l'écrire [la langue] clairement " (GAK 1848-1854, dossier 102).

17 Au terme de l'épreuve, soit on délivrait un diplôme d'aptitude à enseigner, exigé dans l'enseignement privé ${ }^{15}$, soit, dans le cas d'un poste vacant dans la fonction publique, on décidait de la nomination du candidat dans l'enseignement secondaire. Parfois, lorsque le niveau linguistique n'était pas absolument satisfaisant, le jury pouvait même restreindre le champ d'exercice de la profession en orientant le candidat vers une École hellénique, plutôt qu'au Gymnase.

On reste, cependant, perplexe sur les critères présidant à chacun de ces choix car les décisions des commissions sont parfois contredites par celles d'autres jurys constitués pour l'évaluation des manuels scolaires : lorsque Constantin Varvatis, par exemple, déjà titularisé en 1862, soumet sa Grammaire française (1871) au jury constitué pour l'approbation des manuels, on lui reproche un «manque de connaissance exacte du grec et du français» (Antoniou 2012: 117). C'est souvent lorsque ces enseignants deviennent auteurs de manuels que les jurys soulignent leur formation insuffisante, leur manque de méthode d'enseignement ou la faiblesse de leurs ouvrages « rédigés sur des assises désuètes et non conformes aux exigences actuelles » (J.O., $\mathrm{n}^{\circ}$ 119, J.O., fasc. A, 16-5-1909 : 450).

19 Mais devant la pénurie de candidats, il semblerait que les commissions d'évaluation se voient souvent obligées de concéder à la nomination de candidats qui ne satisfont pas toujours tous les critères de sélection. 


\section{Profils d'enseignants}

20 Ce qui précède soulève ainsi une nouvelle série de questions de nature socioprofessionnelle lorsqu'on se penche sur ce nouveau corps enseignant. Qui étaient ces candidats au professorat? De quels milieux provenaient-ils? Quelle était leur formation? Et lorsqu'elle est attestée par des documents officiels, reflète-t-elle la réalité ? Grâce au répertoire biographique élaboré par David Antoniou en 2012, nous disposons aujourd'hui d'un corpus de données importantes permettant de tracer les grandes lignes d'un tableau collectif des enseignants de langue française en Grèce au cours du XIX siècle.

21 Antoniou recense dans son étude 270 individus, dont 163 (60,4\%) sont des Grecs et 107 $(39,6 \%)$ des étrangers. Parmi les Grecs, un bon nombre a reçu une formation privée, d'autres ont fait des études à l'Université othonienne et d'autres en France, ces derniers constituant l'élite du corps professoral. Car, avoir été formé en France est non seulement un atout personnel pour celui qui aspire à entrer dans l'enseignement mais aussi une valeur ajoutée pour l'établissement qui le recrute. D'autres, enfin, ont effectué leurs études dans d'autres pays étrangers tels que l'Allemagne, l'Italie, la Russie ou même la Palestine.

En l'absence d'études universitaires spécialisées, les professeurs de français sont recrutés parmi les diplômés d'autres disciplines : on recense des diplômés de médecine, des juristes ou des théologiens. La combinaison des parcours de formation peut parfois surprendre : médecine et École des ponts et chaussées pour G. Zadès, ou encore École des mines pour I. Soméritis.

23 Nombreux sont ceux qui n'ont pour seules compétences que la connaissance des langues vivantes. Ils connaissent, le français mis à part, l'anglais, l'italien, l'allemand, voire le latin. X. P. Alexandrou est candidat pour trois postes : français, anglais et latin ; G. Politis est professeur d'anglais et auteur d'une chrestomathie anglaise avant de se présenter comme candidat au poste de français. C'est une preuve, sans doute, de l'intérêt que commençaient à avoir les langues vivantes dans le pays. Touchant un autre champ disciplinaire, Foullon enseigne le français, l'italien et les mathématiques. La constatation d'André Chervel sur la polyvalence des professeurs du secondaire au cours du XIX ${ }^{e}$ siècle se confirme aussi dans le cas de la Grèce (Chervel 1988 : 108-109).

24 Être diplômé de l'enseignement supérieur est aussi un atout pour l'obtention d'un poste ou pour la négociation des appointements, comme dans le cas de Hippolyte Teissonière. Étant en plus docteur en droit de l'université de Paris, on apprécie « les connaissances variées, et surtout sa jurisprudence [qui] le mettent à même de se rendre utile aussi d'une autre manière que par le simple enseignement de la langue » (GAK 1833-1848, série 002, dossier 1479 : vue 006).

Les antécédents professionnels se révèlent tout aussi intéressants pour évaluer un milieu professionnel en cours de constitution : un Anglais nommé Black était d'abord policier municipal, puis professeur de langue et littérature anglaises à l'université (GAK 1833-1848, série 002, dossier 931, vue 43) ; G. Vafeiadis qui enseigne le français et le droit commercial est en même temps juge de première instance à Syra. D'autres combinent l'enseignement avec d'autres activités comme la traduction ou le journalisme. 
Bien évidemment, aux données multiples que fournit à la recherche l'ouvrage de Antoniou, il faudrait ajouter le nombre inconnu et difficilement repérable de tous ces francisants qui proposent leurs services dans le secteur privé. Dans leur cas, les petites annonces sont un précieux recours qui permet de révéler des parcours divergents et inégaux qui frisent parfois l'anecdote. Car, le seul fait d'être Français ou d'avoir simplement séjourné en France pendant un certain temps, était considéré comme une formation suffisante pour pouvoir proposer ses services dans le domaine privé.

\section{Enseigner pour se former}

En l'absence, donc, de toute formation ou n'ayant bénéficié que d'une formation approximative, ces premiers professeurs se voient obligés d'improviser et de déployer d'éventuels talents pédagogiques. Mais dans la plupart des cas, le résultat est décevant. Si comme l'avoue G. Zadès dans la préface de sa grammaire, " chaque enseignant avait plus ou moins sa propre méthode d'enseignement" (Zadès 1880: v), les moins talentueux d'entre eux subissaient le rejet, le dédain et les railleries des élèves qui les accueillaient en sifflant et en trépignant, une pratique habituelle dans les écoles grecques du XIX ${ }^{e}$ siècle.

On l'a vu, les commissions d'évaluation ont souvent été contraintes de revoir à la baisse les critères de qualification et ont approuvé la titularisation de candidats qui n'avaient pas les aptitudes requises. De ces derniers, on attend qu'ils comblent leurs lacunes, tant bien que mal, par «la pratique». Les commentaires tirés des comptes rendus des commissions d'évaluation sont autant d'exemples éloquents des lacunes profondes de la formation de certains enseignants ainsi que de leur insuffisance linguistique : « un peu plus de pratique pourra rapidement améliorer ses connaissances sur la langue » (Antoniou 2012: 302); ou encore « sa prononciation présente encore des imperfections, qui se corrigeront par la pratique » (ibid.: 141); « ce n'est que par une pratique de plus longue durée qu'il pourra se familiariser avec la méthode afin de pouvoir être compris de ses élèves » (GAK 1833-1848, série 002, dossier 1530 : vue 57).

29 Formés sur le terrain par la pratique de l'enseignement, un certain nombre de ces professeurs rédige par la suite des manuels. Leurs préfaces contiennent des traces de leur expérience et laissent transparaître leurs conceptions pédagogiques, leurs méthodes d'enseignement expérimentées ou adoptées. Ces mêmes professeurs seront par la suite souvent appelés à constituer à leur tour les commissions de recrutement du personnel enseignant et les jurys d'évaluation des manuels. Ils deviennent, de la sorte, des acteurs de la politique éducative qui vise à uniformiser les pratiques de l'enseignement.

30 Il s'agit d'une période de transition où le maître le plus souvent auto-formé (ce qui n'est pas forcément toujours condamnable) se voit contraint de suivre des directives précises, de respecter un programme préalablement défini par les instances pédagogiques (dont il fait parfois indirectement partie) et d'enseigner la langue à l'aide de manuels préalablement choisis par un jury, ce qui ne manque pas de provoquer des réactions de la part des enseignants. Zadès, se faisant le porte-parole de sa génération, regrette de voir le professeur de français se transformer en un simple fonctionnaire contraint de se plier aux directives du ministère : « chez nous l'enseignant doit se plier, bon gré mal gré, à la méthode et l'ouvrage d'un érudit même si la méthode ou l'ouvrage 
en question sont, comme il a été prouvé pendant quatre ans, les plus défectueux de tous [...] Dans notre pays, l'enseignant doit garder une attitude passive » (Zadès $1888: \mathrm{v}$ ).

\section{Constatations vers la fin du siècle}

31 À la fin du siècle, Athanassios Eftaxias, ministre de l'Instruction, fait le point sur la formation des enseignants du français :

les maîtres et professeurs de langue française chez nous, à quelques rares exceptions près, ont appris la langue de façon empirique, connaissent sa littérature de manière superficielle et, dépourvus de toute méthode, se prêtent plutôt à une parodie d'enseignement et provoquent la compassion et les railleries des élèves. (Eftaxias 1899: 988)

Les dysfonctionnements des classes, le manque de formation des professeurs, les problèmes didactiques préoccupent également les enseignants eux-mêmes qui ressentent le besoin d'un changement. En 1888, Antoine Giannaris, l'un des philologues les plus renommés, formé en Allemagne, et qui occupa différents postes dans l'enseignement, dont celui de professeur de français, explique dans la préface de son manuel intitulé Méthode théorique et pratique pour l'apprentissage de la langue française, que comme nombre de ses confrères, il a dû varier son enseignement et adopter sa propre méthode, car la méthode traditionnelle imposée jusqu'alors par les programmes officiels était, selon lui, désuète et inefficace :

J'ai la conviction inébranlable que l'enseignement des langues étrangères à base de grammaires théoriques et de chrestomathies sans rapport est peine perdue et illusion. L'apprentissage des langues étrangères sans pratique quotidienne est tromperie et chimère. (Giannaris $1888: \mathrm{v}$ )

33 Korpis, un autre de ses confrères, indigné lui aussi des carences du système, revendiquera, en 1904, une formation pour les enseignants et l'introduction de la méthode directe. (Antoniou 2012 : 228-229)

Bien qu'elle fût largement revendiquée, la réorganisation de la discipline se limitera une fois de plus à une révision des critères de sélection des professeurs, qui marque toutefois certains progrès. Eftaxias revoit en effet en 1897 la composition des jurys et définit en détail le contenu des épreuves. Parmi les modifications apportées, notons que l'examen oral et écrit devient obligatoire pour tous les candidats, que dans les jurys siègent désormais des universitaires, ainsi que le directeur de l'École française d'Athènes et un de ses membres, illustration tangible de l'inauguration d'une étroite collaboration franco-hellénique visant à améliorer le niveau des enseignants recrutés (Efthymiou 2013: 47-70). Pour ce qui est du contenu des épreuves, la traduction est conservée à l'écrit (thème et version). Ces exercices sont complétés par une expression écrite à teneur historique, étant donné que les professeurs de français continuent à enseigner l'histoire. L'oral comporte désormais l'explication de texte, l'histoire de la littérature française, la grammaire et la syntaxe mais aussi l'histoire moderne et la géographie (J.0., $\mathrm{n}^{\circ} 117$, fasc. A, 11-8-1897 : 314).

\section{Conclusion}

Cette vue d'ensemble des acteurs professionnels de l'enseignement du français dans la Grèce du XIX ${ }^{e}$ siècle a permis d'observer les premiers efforts de mise en place d'un 
cadre législatif et/ou administratif réglementant les qualifications requises pour les enseignants de français. Avec un peu plus de recul, nous pouvons constater que le professeur de français en Grèce ressemble beaucoup à ses confrères espagnols ou italiens de la même époque (Fernandez Fraile 2005 : 110-119; Mandich 2005 : 63-78). On l'a dit, aucune école, aucun cours ne préparaient ceux qui par vocation ou par besoin se tournaient vers l'enseignement $d u$ français. Ils ne pouvaient perfectionner leurs connaissances qu'à leurs propres frais et au prix d'efforts personnels, où la lecture, principal moyen d'acculturation du XIX siècle, jouait un rôle de première importance. La majorité avait profité de cours particuliers ou de l'enseignement donné dans les écoles congréganistes. Moins nombreux étaient ceux qui avaient les moyens pour effectuer des études à l'étranger. En somme, on attendait de ces professeurs en quelque sorte abandonnés à eux-mêmes qu'ils transmettent aux enfants un savoir-faire à partir d'une culture qu'ils avaient apprise d'une façon empirique. Le très bas niveau de préparation des enseignants explique en outre le peu de considération dont ils jouissaient.

Le profil de l'enseignant de langue française se transforme progressivement au cours du XIX ${ }^{e}$ siècle au gré des modifications de la procédure de sélection. À l'exemple des autres pays européens, on observe en Grèce la volonté étatique de passer à la professionnalisation du métier. Vers la fin du siècle, l'accent n'est plus mis sur l'érudition des individus ou sur leurs divers parcours professionnels mais davantage sur leur formation. Le fait d'être un natif, maîtrisant la langue de son pays, ne suffit plus pour postuler à un poste d'enseignant. Être de nationalité française n'est plus considéré comme un atout exclusif, sauf peut-être pour les écoles privées qui continuent à miser sur cela pour attirer leur clientèle. D'ailleurs, la bonne connaissance du grec continue à être exigée pour la titularisation. Toutefois, si l'on observe ces lentes transformations, le plus grand problème reste toujours présent, à savoir une faible connaissance de la langue malgré une formation qui reste trop théorique et qui se répercute dans l'habileté de parler le français et transmettre ce savoir aux apprenants.

\section{BIBLIOGRAPHIE}

Sources primaires

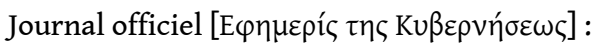

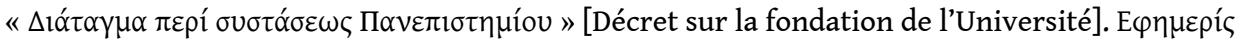

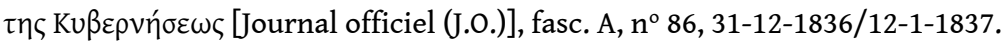

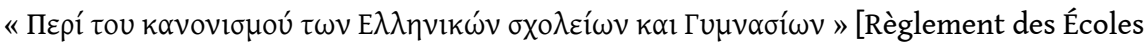
helléniques et des Gymnases]. J.O., fasc. A, nº 87, 31-12-1836/12-1-1837.

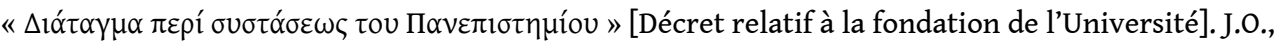
fasc. A, $\mathrm{n}^{\circ} 16,24-4-1837$. 


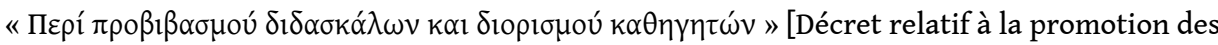
maîtres et la nomination des professeurs]. J.O., fasc. A, $n^{\circ}$ 39, 30-8-1868 : 505.

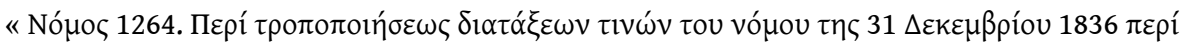

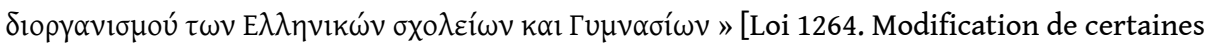
dispositions de la loi du 31-12-1836 sur le règlement des Écoles helléniques et des Gymnases]. J.O., fasc. A, $n^{\circ} 87,31-7-1885: 275-276$.

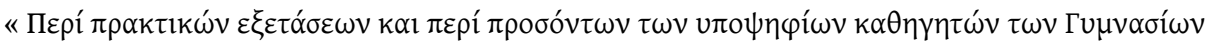
$\kappa \lambda \pi$. » [Sur les examens pratiques et les qualifications des candidats au professorat dans les Gymnases, etc.]. J.O., fasc. A, nº 1, 2-1-1886:1-2.

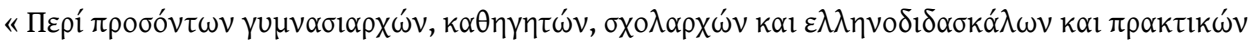

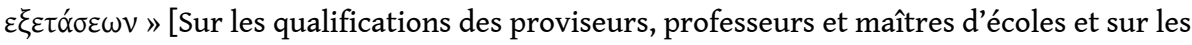
examens pratiques]. J.O., fasc. A, $n^{\circ} 77,12-3-1892: 267-269$.

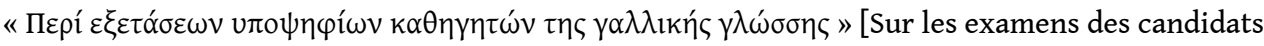
au professorat de français]. J.O., $\mathrm{n}^{\circ}$ 117, fasc. A, 11-8-1897 : 313-314.

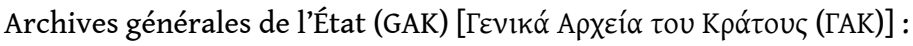

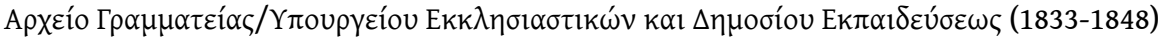

[Archives du secrétariat/ministère des Cultes et de l'Instruction publique (1833-1848)] :

Série 002, dossier 931, vue 43 : Gymnase et École hellénique d'Athènes. En ligne : <http:// arxeiomnimon.gak.gr/browse/resource.html?tab=tab02\&id=10187\&start=20> (consulté le 16/7/2014).

Série 002, dossier 1517 : Progin Soladam. En ligne : <http://srv-gak.att.sch.gr/browse/ resource.html?tab=tab02\&id=523264> (consulté le 16/7/2014).

Série 002, dossier 1530 : Demandeurs de poste et de certificat d'aptitude à l'enseignement. En ligne :

<http://arxeiomnimon.gak.gr/browse/resource.html?tab=tab02\&id=523277\&start=40> (consulté le 16/7/2014).

Série 002, dossier 1526 : Demandeurs de certificat d'aptitude à l'enseignement. En ligne :

<http://arxeiomnimon.gak.gr/browse/resource.html?tab=tab02\&id=523273\&start=440> (consulté le $6 / 7 / 2014)$.

Série 002, dossier 1479 : Hippolyte Teissonière. En ligne : <http://arxeiomnimon.gak.gr/browse/ resource.html?tab=tab02\&id=523220> (consulté le 6/10/2014).

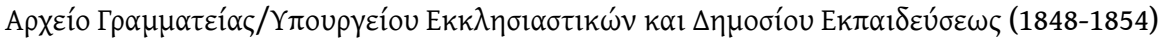

[Archives du secrétariat/ministère des Cultes et de l'Instruction publique (1848-1854)] :

Dossier $82 / 1$ : Loque.

Dossier 100/1 : Marcos Andréadis.

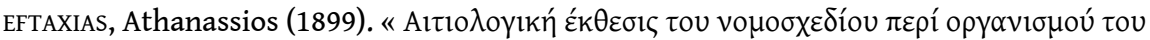

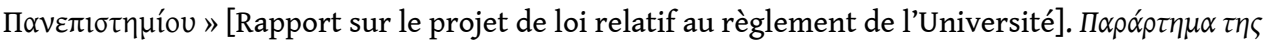

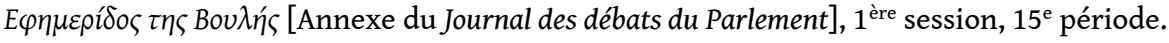
Athènes, 1899 : 950-1003. 


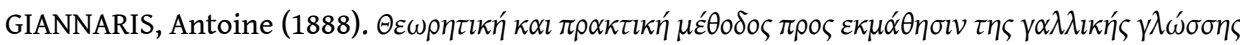
[Méthode théorique et pratique pour l'apprentissage de la langue française]. Athènes : Imprimerie S. K. Vlastos.

[SOLADAM, Progin] (1831). Institution de l'ordre des Solleillans ou chevaliers du soleil. Livourne : chez Jean Zanobetti.

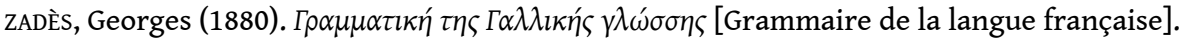

Patras : Sekopoulos.

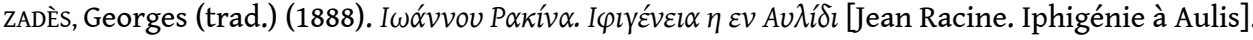
Athènes : Imprimerie Alexandre Papageorgiou.

Sources secondaires

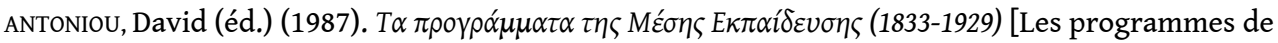
l'enseignement secondaire]. T. 1. Athènes : IAEN.

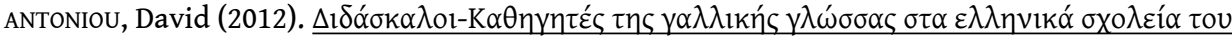

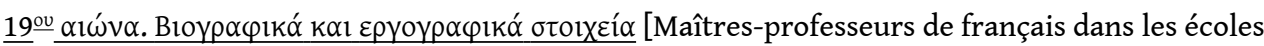
grecques du XIX ${ }^{\mathrm{e}}$ siècle. Éléments biographiques et bibliographiques]. Athènes : CIREL.

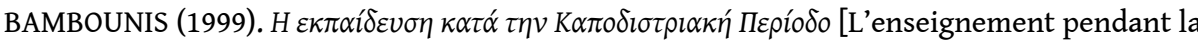
période capodistrienne]. Athènes : Syllogos pros diadosin ophélimon gnoséon.

CHERVEL, André (1988). « L'histoire des disciplines scolaires. Réflexions sur un domaine de recherche ». Histoire de l'éducation, 38, 1988, 59-119.

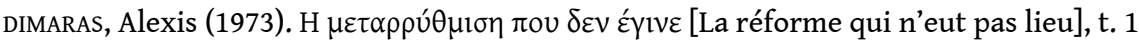

(1821-1894). Athènes : Hermès.

EFTHYMIOU, Loukia (2013). La formation des francisants en Grèce : 1836-1982. Athènes : Éditions Simmetria.

FERNANDEZ FRAILE, Eugenia (2005). « Du maître de langues au professeur : parcours sémantique d'une évolution professionnelle ». In Brigitte Lépinette, María Elena Juménez \& Julia Pinilla (éds), «L'enseignement du français en Europe autour du XIX siècle ». Documents pour l'histoire du français langue étrangère ou seconde, 33/34, 2005, 110-119.

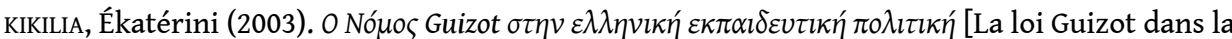
politique éducative grecque]. Thèse de doctorat, Université d'Athènes.

MANDICH, Anna (2005). « Préparation et vérifications des compétences des professeurs de langues vivantes en Italie aux XIX ${ }^{\mathrm{e}}-\mathrm{XX}^{\mathrm{e}}$ siècles ». In Brigitte Lépinette, María Elena Juménez \& Julia Pinilla (éds), « L'enseignement du français en Europe autour du XIX ${ }^{e}$ siècle ». Documents pour l'histoire du français langue étrangère ou seconde, 33/34, 2005, 63-78.

RJÉOUTSKI, Vladislav \& TCHOUDINOV, Alexandre (dir.) (2013). Le précepteur francophone en Europe XVII ${ }^{e}$-XIX ${ }^{e}$ siècles. Paris : L'Harmattan.

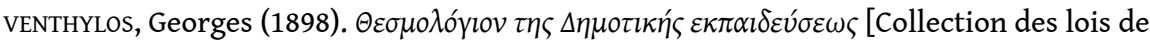
l'enseignement primaire], t. 1. Athènes. 


\section{NOTES}

1. Il s'agit d'une assemblée de notables constituée par les Grecs révoltés du Péloponnèse en mai 1821.

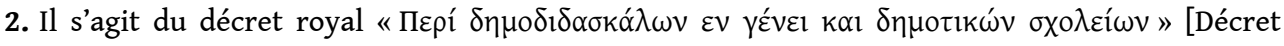
relatif aux instituteurs et aux écoles primaires] promulgué le 6/18 février 1834 (Venthylos 1898 : 10). Cette loi était calquée sur la loi Guizot de 1833 (Kikilia 2003).

3. Selon les divers programmes scolaires appliqués durant le XIXe siècle dans les écoles publiques, la langue française se trouvait très haut dans la hiérarchie des matières enseignées (Antoniou 1987).

4. Nous ne disposons pas d'étude spécifique sur ce phénomène, mais les nombreuses annonces insérées dans la presse hellénique du XIXe siècle confirment la présence de ce groupe socioculturel quantitativement important au sein de la société néohellénique. Sur la présence du précepteur francophone en Europe, on peut consulter l'ouvrage édité par Vladislav Rjéoutski et Alexandre Tchoudinov (2013).

5. Les trois autres étaient la faculté de théologie, la faculté de médecine et la faculté des sciences juridiques et politiques.

6. Il s'agit d'un poste de professeur sans chaire, titre qui fait référence au système universitaire allemand.

7. Le décret prévoit, cependant, la possibilité pour les différentes facultés de solliciter des postes de professeurs extraordinaires, notamment pour les langues vivantes.

8. Afin de justifier la fusion des chaires éventuelles de langues vivantes en une seule chaire de littérature moderne, on ajoute dans la réponse que « la géographie aussi fait aujourd'hui partie de la chaire de l'histoire ".

9. Sur l'histoire de la fondation du département de langue et littérature françaises de l'Université d'Athènes, se reporter à l'ouvrage de Loukia Efthymiou (2013:179-200).

10. Citons à titre d'exemple C. Schinas, N. Vamvas, S. A. Coumanoudis, Efth. Kastorchis et l'historien C. Paparrigopoulos.

11. Comme A. R. Rangabé, auteur de la première chrestomathie française publiée en Grèce.

12. Citons à titre d'exemple Sk. Vyzantios, G. N. Oikonomidis, N. Deloukas, C. Varvatis, I. Carassoutsas et les Français L. Pomerette, H. W. Desrues. Un inventaire systématique des noms qui ont constitué ces différentes commissions pourrait d'ailleurs permettre de mieux cerner les objectifs du concours.

13. On sait, par exemple, qu'en Italie la version proposait un extrait d'un auteur classique et le thème - qui était une épreuve orale - un extrait d'un auteur moderne (Mandich 2005 : 68).

14. Ces évaluations, précieuses aussi pour connaître la constitution des commissions d'évaluation, sont parfois incluses dans les dossiers des candidats au professorat conservés aux Archives générales de l'État (GAK).

15. Il n'est pas rare de voir des candidats ne réclamer que le certificat d'aptitude à l'enseignement. Voir par exemple la demande que fait Constantin Livérios en 1842 (GAK 1833-1848, dossier 1526, vue no 446). 


\section{RÉSUMÉS}

$\mathrm{Au}$ XIXe siècle s'effectue en Grèce l'institutionnalisation progressive de l'enseignement du français langue étrangère. Aussitôt sera posée la question de la formation des professeurs alors qu'aucune structure étatique n'est prévue à cet effet. Si une tentative de créer une chaire universitaire de langue et littérature françaises a échoué, on s'efforce tout au long du XIXe siècle de poser un cadre législatif susceptible de réglementer le recrutement des enseignants. Mais la formation est abandonnée à l'initiative et à la motivation personnelle des enseignants. Formés en France pour ce qui est des plus fortunés d'entre eux, la plupart ont appris la langue en Grèce de façon empirique. Ils forment donc un groupe socio-culturel fort hétérogène. Ce n'est qu'à la fin du siècle que l'on se préoccupe de la réorganisation de la discipline et que l'on pose les jalons pour une formation universitaire qui ne se concrétisera qu'au XXe siècle.

In Greece, in the 19th century Greece, takes place the gradual institutionalization of the teaching of French as a foreign language. At the same time the question of teacher training emerges but the Greek state hasn't provided any official structure for this purpose. An attempt to create a university chair of French language and literature has failed. Only some efforts have been made throughout the 19th century to put a legislative framework in order to regulate the recruitment of teachers. But the teachers training is left to their personal initiative and motivation. The most fortunate among them had been trained in France but the majority have gained the language knowledge by private lessons and through practical understanding in Greece. They thus form a strong diverse socio-cultural group. At the end of the 19th century, we observe a restructuring effort to put the foundations for a university education for the professors of French that will be developed and materialized in the 20th century.

\section{INDEX}

Mots-clés : Grèce, enseignement du français langue étrangère, formation des enseignants, recrutement.

Keywords : Greece, teaching French as a foreign language, teacher training, recruitment.

\section{AUTEUR}

\section{DESPINA PROVATA}

Université nationale et capodistrienne d'Athènes 